\title{
Gambling in Contests with Regret
}

\author{
Han Feng* David Hobson ${ }^{\dagger}$
}

October 31, 2018

\begin{abstract}
This paper discusses the gambling contest introduced in Seel \& Strack [ 6] and considers the impact of adding a penalty associated with failure to follow a winning strategy.

The Seel \& Strack model consists of $n$-agents each of whom privately observes a transient diffusion process and chooses when to stop it. The player with the highest stopped value wins the contest, and each player's objective is to maximise their probability of winning the contest. We give a new derivation of the results of Seel \& Strack [6] based on a Lagrangian approach. Moreover, we consider an extension of the problem in which in the case when an agent is penalised when their strategy is suboptimal, in the sense that they do not win the contest, but there existed an alternative strategy which would have resulted in victory.
\end{abstract}

\section{Introduction}

In [6, Seel \& Strack introduced a model of a gambling contest between agents in which the objective of each agent is not to maximise her return, but rather to maximise the probability that her return is highest amongst the set of agents. One motivation for studying such a problem is that it provides a stylised model for competing fund managers, only the most successful of whom will be given funds to invest over the next time period. Another distinct strand of the literature on modelling competition between fund managers is represented by Basak and Makarov [2].

In the Seel \& Strack paper each agent observes a stochastic process and chooses a stopping time to produce a stopped value. The agent wins the contest if her stopped value is greater than the stopped value of every other agent, and the objective of the agent is to maximise the probability that she wins the contest. Our aim is to add a behavioural finance aspect to the problem. Again the objective of the agent is to maximise her chances of winning the contest,

\footnotetext{
*Department of Statistics, University of Warwick, Coventry, CV4 7AL, UK. H.Feng@warwick.ac.uk

${ }^{\dagger}$ Department of Statistics, University of Warwick, Coventry, CV4 7AL, UK. D.Hobson@warwick.ac.uk
} 
but now she is penalised if she has not won the contest, and she has behaved sub-optimally, in the sense that there was an alternative strategy which would have led to her winning the contest. Thus a fund manager who has followed a poor strategy is not merely given a new role within the firm, but instead is terminated with disgrace.

Although the problem described in [6] is very simple, the solution is remarkably rich and subtle. Firstly, in equilibrium agents must use randomised strategies, so that the level at which the agent should stop is stochastic. Secondly, the set of values at which the agent should stop forms an interval which is bounded above. Several variants are discussed in Seel \& Strack, including the extension to the asymmetric case where the starting values of the processes observed by the agents are different.

We will consider the following variant of the problem. The agent's choice of stopping rule determines her stopped value. But if with hindsight we look at the best possible time she could have chosen then we get a maximum value she might have attained. We consider a problem in which the agent receives a reward of 1 if her stopped value is the highest across all agents, but she is penalised $K$ if her stopped value is not highest, and if she had stopped at the maximum value she might have attained then she would have been the winner. If she is not the winner, and there is no strategy she might have followed which would led to her being the winner then her score is zero.

In fact we consider three variants of the problem, in which an omniscient being (or the agent's supervisor) penalises the agent for stopping too soon, for stopping too late, or for stopping too soon or too late. In the first case the agent faces regret over stopping too soon, and we consider the maximum value to be the maximum value attained by her process after the moment she chose to stop. In the second case the maximum is taken only over that part of the path which occurs before the chosen stopping time and the agent faces regret over stopping too late. In the third case we take the maximum over the whole path.

Our results are that in the first problem, the effect of the penalty is precisely equivalent to an increase in the number of opponents. An increase in $K$ incentives the agent to aim for higher values, at the cost of stopping at low values more often. This is the same as the effect of competition from more opposing agents. In the second problem, which is both harder and more interesting, the optimal strategy is modified in a more subtle way. This case is relevant if the agent's process is unobservable from the point at which is is stopped, for instance if it is the gains from trade process arising from a dynamic investment strategy chosen by the agent. Now the agent faces a risk of a penalty whenever she stops below the value of the current maximum. For this reason she is reluctant to do so, although it is also sub-optimal to wait until her process hits zero, as this is a sure losing strategy. An increase in $K$ incentives her to stop more quickly. The third problem might be expected to be a combination of the two previous problems, but in fact there is a natural simplification which leads to the optimum being the same solution as the original Seel \& Strack problem.

The remainder of this paper is constructed as follows. In the next section we describe the original contest introduced in Seel \& Strack [6]. We rederive 
the Nash equilibrium, using a different approach based on Lagrangian method. Then in Section 3 we introduce the problem with regret, which we then solve in the three cases described above in Sections 4, 5 and 6. Finally in Section 7 we explain the origin of the optimal multipliers and the candidate Nash equilibrium distribution. The ideas of the proofs are different to those in [6] in that instead of trying to write down the value function for the agents we use a Lagrangian sufficiency theorem. This brings new insights and yields a simpler proof even in the standard problem of Seel \& Strack and facilitates our analysis in the extended problem.

\section{Contest without regret}

\subsection{The model}

There are $n$ players with labels $i \in I=\{1,2, \ldots, n\}$ who take part in the contest. Player $i$ privately observes the continuous-time realisation of a Brownian motion $X^{i}=\left(X_{t}^{i}\right)_{t \in \mathbb{R}^{+}}$absorbed at zero with $X_{0}^{i}=x_{0}$ where $x_{0}$ is independent of $i$ and positive. Let $\mathcal{F}_{t}^{i}=\sigma\left(\left\{X_{s}^{i}: s<t\right\}\right)$ and set $\mathbb{F}^{i}=\left(\mathcal{F}_{t}^{i}\right)_{t \geq 0}$.

The space of strategies for agent $i$ is the space of $\mathbb{F}^{i}$-stopping times $\tau^{i}$. Since zero is absorbing for $X^{i}$, without loss of generality we may restrict attention to $\tau^{i} \leq H_{0}^{i}=\inf \left\{t \geq 0: X_{t}^{i}=0\right\}$. Player $i$ observes her own process $X^{i}$, but not $X^{j}$ for $j \neq i$; nor does she observe the stopping times chosen by the other agents. Moreover, the processes $X^{i}$ are independent.

The player who stops at the highest value wins unit reward, that is, $\forall i \in I$, player $i$ wins 1 if she stops at time $\tau^{i}$ such that $X_{\tau^{i}}^{i}>X_{\tau^{j}}^{j} \forall j \neq i$. If there are $k$ players who stop at the equal highest value then these players each win $\frac{1}{k}$. Therefore player $i$ with stopping value $X_{\tau^{i}}^{i}$ receives payoff

$$
\frac{1}{k} \mathbf{1}_{\left\{X_{\tau^{i}}^{i}=\max _{j \in I} X_{\tau^{j}}^{j}\right\}},
$$

where $k=\left|\left\{i \in I: X_{\tau^{i}}^{i}=\max _{j \in I} X_{\tau^{j}}^{j}\right\}\right|$.

The key insight of Seel \& Strack [ 6 is to observe that the problem of choosing the optimal stopping time can be reduced to a problem of finding the optimal law for $X_{\tau}$ or equivalently an optimal target distribution. The payoffs to the agents only depend upon $\tau^{i}$ via the distribution of $X_{\tau^{i}}^{i}$. Hence, the problem can be considered in two stages, firstly find an optimal target distribution $F^{i}$, and then verify that there is a choice of $\tau^{i}$ such that $X_{\tau^{i}}^{i}$ has law $F^{i}$. But, the problem of finding $\tau$ such that $X_{\tau}$ has law $F$ is a classical problem in probability theory, and is known as the Skorokhod embedding problem (Skorokhod [7]). Since $X$ is a Brownian motion started at $x_{0}$ and absorbed at 0 , any distribution on $\mathbb{R}^{+}$ with mean less than or equal to $x_{0}$ can be embedded with a finite stopping time $\tau$ (and conversely, for any $\tau$ the law of $X_{\tau}$ has mean less than or equal to $x_{0}$ ).

Note that there are multiple solutions to the Skorokhod embedding problem for $F$, so there will be several alternative stopping rules which will bring equal 
probability of success to an agent. However, we expect that the optimal target distribution is unique.

Our aim is to find Nash equilibria for the problem. By the above remarks, a Nash equilibria can be identified with a family of distribution functions $\left(F^{i}\right)_{i \in I}$. We will say that $\left(F^{i}\right)_{i \in I}$ is a Nash equilibrium if, for each $i \in I$, if the other agents use stopping rules $\tau^{j}$ such that $X_{\tau^{j}}^{j} \sim F^{j}$, then the optimal target distribution for agent $i$ is $F^{i}$, and she may use any stopping rule $\tau^{i}$ such that $X_{\tau^{i}}^{i} \sim F^{i}$. We will say a Nash equilibrium is symmetric if $F^{i}$ does not depend on $i$, and we will say that a Nash equilibrium is atom-free if each $F^{i}$ is atom-free. Given the symmetry of the situation in the sense that each agent observes a martingale process started from the same level $x_{0}$, it seems natural that a Nash equilibria is symmetric. Moreover, simple arguments over rearranging mass can be used to show that it is never optimal for two agents to put mass at the same positive point $x$ - either of them could benefit by modifying the target distribution to put a proportion $N /(N+1)$ of this mass at $\left(x+N^{-2}\right)$ and a proportion $1 /(N+1)$ at $\left(x-N^{-1}\right)$ - and then it is possible to deduce that any optimal solution is atom-free.

Theorem 2.1. [Seel $\mathscr{E}$ Strack [6]] Any Nash equilibrium has the property that it is symmetric and atom-free.

Remark 2.1. The fact that the Nash equilibria is atom-free relies on the fact that the situation is symmetric in the sense that all agents stop Brownian motions started from a common value $x_{0}$. If the agents observe processes with different starting points, then the Nash equilibria may have masses at zero for some agents. In that case, for a Nash equilibrium, no agent places mass at a positive point, and at least one agent has an atom-free distribution. We will only consider the symmetric case.

Remark 2.2. In the Seel \& Strack setting, which we call the standard case, we will focus on proving that there exists a unique symmetric, atom-free Nash equilibria. It will then follow from the results of Seel \& Strack [6] that this is the unique Nash equilibrium for our problem. Our methods can be extended to show that every Nash equilibrium has the property that it is symmetric and atom-free, but we will not present those arguments here. When we consider the problem with a penalty for using a losing strategy when a winning strategy exists, we will again prove the existence of a unique symmetric atom-free Nash equilibrium, but intuition gained from the standard case indicates that this equilibrium is unique.

\subsection{Derivation of the equilibrium distribution}

This section is devoted to a proof of the following result, first proved in Seel and Strack [6] using different methods.

Theorem 2.2. There exists a symmetric, atom-free Nash equilibrium for the 
problem for which $X_{\tau^{i}}^{i}$ has law $F(x)$, where for $x \geq 0$

$$
F(x)=\min \left\{\sqrt[n-1]{\frac{x}{n x_{0}}}, 1\right\}
$$

Proof. Let $\mathcal{A}$ be the set of non-decreasing functions $f:[0, \infty) \mapsto[0, \infty)$ which are null at zero, and let $\mathcal{A}_{D}\left(x_{0}\right)$ be the subset of $\mathcal{A}$ corresponding to distribution functions of random variables with mean $x_{0}$. Then,

$\mathcal{A}_{D}\left(x_{0}\right)=\left\{f:[0, \infty) \mapsto[0, \infty)\right.$ such that $f(0)=0, f$ non-decreasing, $\left.\lim _{x \uparrow \infty} f(x)=1, \int_{0}^{\infty} x f(d x)=x_{0}\right\}$.

We seek a symmetric atom-free Nash equilibrium. Since there are no atoms, we do not need to consider how to break ties and a symmetric Nash equilibrium is identified with a distribution function $G^{*} \in \mathcal{A}_{D}\left(x_{0}\right)$ with the property that

$$
\int_{0}^{\infty} G^{*}(x)^{n-1} G^{*}(d x) \geq \int_{0}^{\infty} G^{*}(x)^{n-1} G(d x) \quad \forall G \in \mathcal{A}_{D}\left(x_{0}\right) .
$$

Suppose that the other players all choose $F(x)$ as their target distribution. Then the problem facing the agent is to choose $G$ to solve

$$
\max _{G(x) \in \mathcal{A}} \int_{0}^{\infty} F(x)^{n-1} G(d x) \text { subject to } \int_{0}^{\infty} x G(d x)=x_{0} \text { and } \int_{0}^{\infty} G(d x)=1 .
$$

Introducing multipliers $\lambda$ and $\gamma$ for the two constraints, the Lagrangian for the optimization problem (1) is then

$$
\mathcal{L}_{F}(G ; \lambda, \gamma)=\int_{0}^{\infty}\left[F(x)^{n-1}-\lambda x-\gamma\right] G(d x)+\lambda x_{0}+\gamma .
$$

Now we state a variant of the Lagrangian sufficiency theorem for our problem.

Proposition 2.1. If $G^{*}, \lambda^{*}$ and $\gamma^{*}$ exist such that $G^{*} \in \mathcal{A}_{D}\left(x_{0}\right)$ and

$$
\mathcal{L}_{G^{*}}\left(G^{*} ; \lambda^{*}, \gamma^{*}\right) \geq \mathcal{L}_{G^{*}}\left(G ; \lambda^{*}, \gamma^{*}\right)
$$

for all $G \in \mathcal{A}$, then $G^{*}$ is a symmetric, atom-free Nash equilibrium.

Proof. If $G \in \mathcal{A}_{D}\left(x_{0}\right)$ then

$$
\int_{0}^{\infty} G^{*}(x)^{n-1} G(d x)=\mathcal{L}_{G^{*}}\left(G ; \lambda^{*}, \gamma^{*}\right) .
$$

Then, under the hypotheses of the proposition,

$$
\int_{0}^{\infty} G^{*}(x)^{n-1} G^{*}(d x)=\mathcal{L}_{G^{*}}\left(G^{*} ; \lambda^{*}, \gamma^{*}\right) \geq \mathcal{L}_{G^{*}}\left(G ; \lambda^{*}, \gamma^{*}\right)=\int_{0}^{\infty} G^{*}(x)^{n-1} G(d x) .
$$


Return to the proof of Theorem 2.2. On $[0, \infty)$ let $G^{*}(x)=\min \left\{\sqrt[n-1]{x /\left(n x_{0}\right)}, 1\right\}$, $\lambda^{*}=1 /\left(n x_{0}\right)$ and $\gamma^{*}=0$. We verify that for these multipliers (2) holds and that $G^{*} \in \mathcal{A}_{D}\left(x_{0}\right)$. The latter follows immediately from the explicit form of $G^{*}$. For the former

$$
\begin{aligned}
\mathcal{L}_{G^{*}}\left(G ; \lambda^{*}, \gamma^{*}\right) & =\int_{0}^{\infty}\left[G^{*}(x)^{n-1}-\lambda^{*} x-\gamma^{*}\right] G(d x)+\lambda^{*} x_{0}+\gamma^{*} \\
& =\int_{n x_{0}}^{\infty}\left[1-\frac{x}{n x_{0}}\right] G(d x)+\frac{1}{n} \leq \frac{1}{n}=\mathcal{L}_{G^{*}}\left(G^{*} ; \lambda^{*}, \gamma^{*}\right) .
\end{aligned}
$$

Thus there exists a symmetric, atom-free Nash equilibrium of the given form.

Remark 2.3. Seel \& Strack [6] solve the problem by writing down a candidate value function for the problem, and then verifying that the candidate value function is a martingale under an optimal stopping rule for each agent.

Remark 2.4. Our results can be extended to the case where the processes observed by the agents are independent copies of some time-homogeneous diffusion process $Y$ which converges almost surely to the lower bound on its state space. The idea is to use a change of scale, and, in the setting of Skorokhod embeddings, can be traced back to Azéma and Yor [1. In addition to Brownian motion (absorbed at zero), canonical examples include exponential Brownian motion and drifting Brownian motion with negative drift (with or without absorption at zero). Seel \& Strack [6] consider the problem when $Y^{i}$ is a Brownian motion with positive drift, absorbed at zero, but then they need to impose a further condition on the model parameters to ensure the finiteness of the candidate solution.

Let the state space of the time-homogeneous diffusion $Y$ be an interval $S$ with endpoints $\{l, r\}$ with $-\infty \leq l<Y_{0}=y_{0}<r \leq \infty$. Suppose that $Y$ is a solution of the stochastic differential equation $d Y=a(Y) d B+b(Y) d t$ and let $s=s(y)$ be the scale function 1 . Then $s$ is an increasing solution of $a(y)^{2} s^{\prime \prime}(y)+2 b(y) s^{\prime}(y)=0$ and $X=s(Y)$ is a continuous local martingale with starting value $x_{0}=s\left(y_{0}\right)$, and hence a time-change of Brownian motion. Moreover, our assumption that $Y$ converges to the lower boundary implies that $s(l)$ is finite whereas $s(r)=\infty$ and without loss of generality we may set $s(l)=0$. Then $X=s(Y)$ converges to zero almost surely (and if zero can be reached in finite time, then zero is absorbing).

Note that $s(\cdot)$ is a continuous strictly increasing function. Hence the payoff of player $i$ with stopping value $Y_{\tau^{i}}^{i}$ can be expressed as $\frac{1}{k} \mathbf{1}_{\left\{X_{\tau^{i}}^{i}=\max _{j \in I} X_{\tau_{j}^{j}}^{j}\right\}}$, where $k=\left|\left\{i \in I: X_{\tau^{i}}^{i}=\max _{j \in I} X_{\tau^{j}}^{j}\right\}\right|$, and $X_{\tau^{i}}^{i}=s\left(Y_{\tau^{i}}^{i}\right)$. Then the contest in which players privately observe $Y^{i}$ is equivalent to the contest in which players

\footnotetext{
${ }^{1}$ If $Y$ is exponential Brownian motion, $d Y=a Y d B+b Y d t$ then $s(y)=y^{\kappa}$ with $\kappa=$ $1-2 b / a^{2}$. Note that we need parameters such that $\kappa>0$ to ensure that $Y$ is transient to zero. If $Y$ is downward drifting Brownian motion, $d Y=a d B+b d t$ with $b<0$, then $s(y)=e^{-\eta y}$ with $\eta=2 b / a^{2}$.
} 
privately observe $X^{i}$, and the choice of the optimal $\tau^{i}$ is the same for both problems. In particular, if we have a Nash equilibrium for which $\tau^{i}$ is optimal for the processes $X^{i}$, then we also have a Nash equilibrium for the processes $Y^{i}$.

The problem is then to find a Nash equilibrium $\left(G^{i}\right)_{i \in I}$ for $Y_{\tau^{i}}^{i}$ and then verify that there exists $\tau^{i}$ such that $Y_{\tau^{i}}^{i}$ has law $G^{i}$. Under our transformation, this is the same as finding a Nash equilibrium $\left(F^{i}\right)_{i \in I}$ for $X_{\tau^{i}}^{i}$ where $F^{i}=$ $G^{i} \circ s^{-1}$, where $s^{-1}$ is the inverse of $s$. To solve the problem for $X$, then either we argue that the only properties of $X$ that we use are the strong Markov property, the local martingale property, and the fact that $X$ converges to zero, so that the theory of this section applies to the local martingale diffusion $X$, or we argue that since $X$ is a non-negative martingale diffusion, $X$ is a time-change of Brownian motion and $X_{t}=B_{\Gamma_{t}}$ for some increasing functional $\Gamma_{t}$. Then if $F$ is any distribution with mean less than or equal to $x_{0}$, and $\sigma$ is a stopping time such that $B_{\sigma} \sim F$, then we may take $\tau=\Gamma^{-1} \circ \sigma$ and then $X_{\tau}=B_{\sigma} \sim F$ and $\tau$ is an embedding of $G$ in $Y$.

Note that if $X_{\tau} \sim F$ and $F$ has mean $x_{1}<x_{0}$, then there exists $(\tilde{F}, \tilde{\tau})$ such that $\tilde{F}$ has mean $x_{0}, \tilde{F} \geq F$ and $X_{\tilde{\tau}} \sim \tilde{F}$. Clearly $\tilde{\tau}$ dominates $\tau$ as a strategy. Hence we may restrict attention to stopping times $\tau$ such that the distribution $F$ of $X$ has mean $x_{0}$, and then $\left(X_{t \wedge \tau}\right)_{t \geq 0}$ is a martingale and not just a local martingale.

\section{Contests with regret}

Our goal is to solve an extended version of the problem in which agents are penalised for following losing strategies, if they had an alternative stopping rule which would have won the contest. The idea is that there is an omniscient judge who can observe the path of $X^{i}$, and not just the stopped value, and who penalises the agent for the failure to use a winning stopping rule if such a strategy exists. This judge represents the supervisor of the agent, and the agent faces penalties (such as dismissal) in cases where after the fact she is seen to have followed a losing strategy, when a winning strategy existed.

In our model there are $n$ contestants, each of whom privately observes their own process $X^{i}$ and the player with the highest stopping value wins unit reward. That is, $\forall i \in I$, player $i$ wins 1 if she stops at time $\tau^{i}$ such that $X_{\tau^{i}}^{i}>X_{\tau^{j}}^{j}$ $\forall j \neq i$. In addition the player is penalised $K \geq 0$ if her stopped value is not highest, and if she had an alternative strategy which would, with the benefit of hindsight, have allowed her to win. (The case $K=0$ corresponds to the standard problem.) Given that the best strategy for agent $i$ is to stop at the maximum value $M^{i}$ attained by $X^{i}$ this means that player $i$ loses $K$ if she stops at $\tau^{i}$ such that $X_{\tau^{i}}^{i}<\max _{j \neq i} X_{\tau^{j}}^{j}<M^{i}$.

As before, ties are broken randomly. If there are $k$ players who stop at the highest value then these players each wins $\frac{1}{k}$. Further, player $i$ loses $K_{2}$ if she stops at $\tau^{i}$ such that $X_{\tau^{i}}^{i}<\max _{j \neq i} X_{\tau^{j}}^{j}=M^{i}$, where $0 \leq K_{2} \leq K$. Hence 
player $i$ who stops at $X_{\tau^{i}}^{i}$ with maximum value $M^{i}$ has payoff

$$
\frac{1}{k} \mathbf{1}_{\left\{X_{\tau^{i}}^{i}=\max _{j \in I} X_{\tau^{j}}^{j}\right\}}-K \mathbf{1}_{\left\{X_{\tau^{i}}^{i}<\max _{j \neq i} X_{\tau^{j}}^{j}<M^{i}\right\}}-K_{2} \mathbf{1}_{\left\{X_{\tau^{i}}^{i}<\max _{j \neq i} X_{\tau^{j}}^{j}=M^{i}\right\}},
$$

where $k=\left|\left\{i \in I: X_{\tau^{i}}^{i}=\max _{j \in I} X_{\tau^{j}}^{j}\right\}\right|$.

Our objective is to find a Nash equilibria which is represented by a family of stopping rules $\left(\tau^{i}\right)$. Since the values $\left(X_{\tau^{i}}^{i}, M^{i}\right)$ are a sufficient statistic for the problem, the Nash equilibria can be characterised by the law $\nu^{i}$ of $\left(X_{\tau^{i}}^{i}, M^{i}\right)$. Then in equilibrium, the agent can use any stopping rule for which $\left(X_{\tau^{i}}^{i}, M^{i}\right)$ has law $\nu^{i}$. We write $F^{i}$ for the marginal of $\nu$ which corresponds to the law of $X_{\tau^{i}}^{i}$.

In the standard case, every Nash equilibrium is symmetric and atom-free. In our generalised setting we will limit our search to symmetric atom-free Nash equilibria, and we will show that there exists a unique such equilibrium. Motivated by the situation in the standard case we conjecture that every Nash equilibria is symmetric and atom-free and therefore that we have found the unique equilibrium.

Remark 3.1. Since there are no atoms, the probability of a tie is zero. Thus neither the method of breaking ties nor the value of $K_{2}$ will affect our results.

Suppose that the other players all choose $F(x)$ as their target distribution of $X_{\tau}$. Then agent $i$ aims to choose a feasible measure $\nu(x, y)$ for $\left(X_{\tau^{i}}^{i}, M^{i}\right)$ to maximise

$$
\begin{aligned}
\mathbb{E}\left[F\left(X_{\tau^{i}}^{i}\right)^{n-1}\right] & -K \mathbb{E}\left[F\left(M^{i}\right)^{n-1}-F\left(X_{\tau^{i}}^{i}\right)^{n-1}\right] \\
& =(1+K) \mathbb{E}\left[F\left(X_{\tau^{i}}^{i}\right)^{n-1}\right]-K \mathbb{E}\left[F\left(M^{i}\right)^{n-1}\right], \\
& =\int_{0}^{\infty} \int_{0}^{\infty}\left[(1+K) F(x)^{n-1}-K F(y)^{n-1}\right] \nu(d x, d y),
\end{aligned}
$$

which is her expected payoff when she stops at $\tau^{i}$.

So far we have been imprecise about the definition of $M^{i}$. The quantity $M^{i}$ represents the maximum the agent could have achieved. Depending on the interpretation, this could be the maximum over the entire path $M^{i}=\max \left\{X_{t}^{i} ; 0 \leq\right.$ $\left.t \leq H_{0}^{i}\right\}$, or it could be that only that part of the path before the agent's chosen stopping time is considered, $M^{i}=\max \left\{X_{t}^{i} ; 0 \leq t \leq \tau^{i}\right\}$, or only that part of the path after the agent's chosen stopping time, $M^{i}=\max \left\{X_{t}^{i} ; \tau^{i} \leq t \leq H_{0}^{i}\right\}$, where $H_{0}^{i}=\inf \left\{t \in \mathbb{R}^{+}: X_{t}^{i}=0\right\}$. These different interpretations will lead to different Nash equilibria. We consider the three cases separately in the next three sections.

\section{Contest with regret over future failure to stop}

In this section we consider the contest in which the agent is penalised for stopping too soon. We consider the maximum value $M^{i}$ to be defined by

$$
M^{i}:=M_{\left[\tau^{i}, H_{0}^{i}\right]}^{i}=\sup _{\tau^{i} \leq t \leq H_{0}^{i}} X_{t}^{i} .
$$


Theorem 4.1. There exists a symmetric, atom-free Nash equilibrium for the problem for which $X_{\tau^{i}}^{i}$ has law $F(x)$, where for $x \geq 0$

$$
F(x)=\min \left\{\sqrt[N-1]{\frac{x}{N x_{0}}}, 1\right\}
$$

with $N=n+K(n-1)$.

Remark 4.1. The agent follows exactly the same optimal strategy as an agent in a different setup, where there is no penalty, but the total number of contestants is increased to $N=n+K(n-1)$.

Proof. Denote by $\nu$ the joint distribution of $X_{\tau^{i}}^{i}$ and $M_{\left[\tau^{i}, H_{0}^{i}\right]}^{i}$ and denote by $G(x)$ the marginal distribution of $X_{\tau^{i}}^{i}$. Then using the strong Markov property and the martingale property of $X$,

$$
\begin{aligned}
\nu([0, x] \times[0, y]) & =\mathbb{P}\left(X_{\tau^{i}}^{i} \leq x, M_{\left[\tau^{i}, H_{0}^{i}\right]}^{i} \leq y\right)=\int_{0}^{x} \mathbb{P}\left(M_{\left[\tau^{i}, H_{0}^{i}\right]}^{i} \leq y \mid X_{\tau^{i}}^{i}=z\right) G(d z) \\
& =\int_{0}^{x} \mathbb{P}\left(H_{0}^{i}<H_{y}^{i} \mid X_{0}^{i}=z\right) G(d z)=\int_{0}^{x} \frac{y-z}{y} G(d z),
\end{aligned}
$$

where $H_{y}^{i}=\inf \left\{t \in \mathbb{R}^{+}: X_{t}^{i}=y\right\}$.

Suppose that the other players all choose $F(x)$ as their target distribution of $X_{\tau}$. Substituting (4) into (3), the expected payoff of player $i$ becomes

$$
\begin{aligned}
\int_{0}^{\infty} \int_{0}^{\infty} & {\left[(1+K) F(x)^{n-1}-K F(y)^{n-1}\right] \nu(d x, d y) } \\
= & \int_{0}^{\infty}(1+K) F(x)^{n-1} G(d x)-\int_{0}^{\infty} \int_{x}^{\infty} K F(y)^{n-1} \frac{x}{y^{2}} d y G(d x) \\
= & \int_{0}^{\infty}\left[(1+K) F(x)^{n-1}-K x \int_{x}^{\infty} \frac{F(y)^{n-1}}{y^{2}} d y\right] G(d x) .
\end{aligned}
$$

Given other players' choices, player $i$ would like to choose $G \in \mathcal{A}$ to solve $\max _{G \in \mathcal{A}} \int_{0}^{\infty}\left[(1+K) F(x)^{n-1}-K x \int_{x}^{\infty} \frac{F(y)^{n-1}}{y^{2}} d y\right] G(d x)$ subject to $\int_{0}^{\infty} x G(d x)=x_{0}$ and $\int_{0}^{\infty} G(d x)=1$

Introducing multipliers $\lambda$ and $\gamma$ for the two constraints, the Lagrangian for the optimization problem (5) is then

$\mathcal{L}_{F}(G ; \lambda, \gamma)=\int_{0}^{\infty}\left[(1+K) F(x)^{n-1}-K x \int_{x}^{\infty} \frac{F(y)^{n-1}}{y^{2}} d y-\lambda x-\gamma\right] G(d x)+\lambda x_{0}+\gamma$.

On $[0, \infty)$ let $G^{*}(x)=\min \left\{1, \sqrt[N-1]{x /\left(N x_{0}\right)}\right\}, \lambda^{*}=1 /\left(N x_{0}\right)$ and $\gamma^{*}=0$, where $N=n+K(n-1)$. It is easy to check that $G^{*} \in \mathcal{A}_{D}\left(x_{0}\right)$. Moreover,

$$
\begin{aligned}
\mathcal{L}_{G^{*}}\left(G ; \lambda^{*}, \gamma^{*}\right) & =\int_{0}^{\infty}\left[(1+K) G^{*}(x)^{n-1}-K x \int_{x}^{\infty} \frac{G^{*}(y)^{n-1}}{y^{2}} d y-\lambda^{*} x-\gamma^{*}\right] G(d x)+\lambda^{*} x_{0}+\gamma^{*} \\
& =\int_{N x_{0}}^{\infty}\left[1-\frac{x}{N x_{0}}\right] G(d x)+\frac{1}{N} \leq \frac{1}{N}=\mathcal{L}_{G^{*}}\left(G^{*} ; \lambda^{*}, \gamma^{*}\right) .
\end{aligned}
$$


Hence, by the Lagrangian sufficiency theorem (Proposition 2.1) $G^{*}$ is a symmetric, atom-free Nash equilibrium.

Remark 4.2. In this version of the problem, the stopping decision depends on the current value of $X$ alone, and not on the current maximum. This is because the penalty depends on the future maximum, which conditional on the current value of the process is independent of the past maximum.

\section{Contest with regret over past failure to stop}

This section discusses the contest with regret over past failure to stop, that is player is penalised when she could have won if she had stopped sooner. This case is relevant when the omniscient being can only observe the realisation of $X^{i}$ up to the stopping time chosen by the agent. In this case the maximum value $M^{i}$ is defined by

$$
M^{i}:=M_{\tau^{i}}^{i}=\sup _{0 \leq t \leq \tau^{i}} X_{t}^{i}
$$

Consider the problem facing a single agent under the assumption that the strategies of the competing agents are fixed. Temporarily we drop the subscript denoting the label of the agent. Recall that the payoff to the agent is $(1+$ $K) F\left(X_{\tau}\right)^{n-1}-K F\left(M_{\tau}\right)^{n-1}$. For a continuous martingale Kertz and Rösler [4] characterise all possible joint laws of $\left(X_{\tau}, M_{\tau}\right)$ and hence the problem is reduced to a search over measures with these characteristics. However, an alternative is to split the optimisation problem into a two-stage procedure: first for any feasible distribution of $X_{\tau}$ (a non-negative random variable with mean $x_{0}$ ) find the joint law of $\left(X_{\tau}, M_{\tau}\right)$ for which $M_{\tau}$ is as small as possible in distribution such a joint law exists by results of Perkins [5] - and then minimise a modified objective function over feasible laws of $X_{\tau}$.

For a given law of $X_{\tau}$ the joint law of $\left(X_{\tau}, M_{\tau}\right)$ for which $M_{\tau}$ is minimised is such that mass is placed only on the set $A=\left\{(x, x) ; x \geq x_{0}\right\} \cup\{(x, \Phi(x)) ; x<$ $\left.x_{0}\right\}$ where $\Phi:\left(0, x_{0}\right) \mapsto\left(x_{0}, \infty\right)$ is a decreasing function (and if $X_{\tau}$ is atom-free, a strictly decreasing function). Let $\phi$ be inverse to $\Phi$. Then, if $G$ denotes the marginal law of $X$, we can conclude from Doob's submartingale inequality 2 , in conjunction with the set identity $\left(M_{\tau} \geq m\right)=\left(X_{\tau} \geq m\right) \cup\left(X_{\tau} \leq \phi(m)\right)$, that for $m \geq x_{0}$

$0=\mathbb{E}\left[m-X_{\tau} ; X_{\tau} \geq m\right]+\mathbb{E}\left[m-X_{\tau} ; X_{\tau} \leq \phi(m)\right]=\int_{m}^{\infty}(m-y) G(d y)+\int_{0}^{\phi(m)}(m-y) G(d y)$

which, since $X_{\tau}$ has mean $x_{0}$, is equivalent to

$$
0=m-x_{0}+(m-\phi(m)) G(\phi(m))-\int_{\phi(m)}^{m} G(y) d y .
$$

\footnotetext{
${ }^{2}$ By the final remark of Section 2.2 we may assume $\left(X_{t \wedge \tau}\right)_{t \geq 0}$ is a martingale, and then we have, for $m \geq x_{0}, m \mathbb{P}\left(M_{\tau} \geq m\right)=\mathbb{E}\left[X_{\tau} ; M_{\tau} \geq m\right]$. Hence $0=\mathbb{E}\left[m-X_{\tau} ; M_{\tau} \geq m\right]$.
} 
In differential form, assuming $G$ and $\phi$ are differentiable, this becomes

$$
0=\phi^{\prime}(m)(m-\phi(m)) G^{\prime}(\phi(m))+1+G(\phi(m))-G(m) .
$$

It follows from the results of Perkins [5] and Hobson and Pedersen [3], that if $G$ is the law of an atom-free non-negative random variable, then there exists a decreasing function $\phi$ solving (6). Further, if $\xi$ is a random variable such that for $s \geq x_{0}$

$$
\mathbb{P}(\xi \geq s)=\exp \left(-\int_{\left(x_{0}, s\right)} \frac{G(d u)}{1-G(u)+G(\phi(u))}\right)
$$

and if $\tau=\tau_{\xi} \wedge \tau_{\phi}$ where $\tau_{\xi}=\inf \left\{t>0 \mid M_{t} \geq \xi\right\}$ and $\tau_{\phi}=\inf \left\{t>0 \mid X_{t} \leq\right.$ $\left.\phi\left(M_{t}\right)\right\}$, then $X_{\tau}$ has law $G$ and $\left(X_{\tau}, M_{\tau}\right)$ places no mass off $A$. Moreover, amongst the class of joint laws for $\left(X_{\tau}, M_{\tau}\right)$ such that $X_{\tau}$ has law $G, M_{\tau}$ is as small as possible in distribution.

Theorem 5.1. Suppose there exists a finite real number $r>x_{0}$, a once differentiable strictly decreasing function $\phi:\left[x_{0}, r\right] \mapsto\left[0, x_{0}\right]$, a thrice differentiable strictly increasing and strictly convex function $\psi:\left[x_{0}, r\right] \mapsto[0,1]$ and a once differentiable strictly decreasing function $\theta:\left[x_{0}, r\right] \mapsto[0,1]$ such that $\phi, \psi$ and $\theta$ solve the following system of equations

$$
(\dagger)\left\{\begin{array}{l}
\phi^{\prime}(y) \psi^{\prime}(y)=(1+K) \theta^{\prime}(y), \\
K \psi^{\prime}(y)=(y-\phi(y)) \psi^{\prime \prime}(y), \\
\frac{y-\phi(y)}{n-1} \theta^{\prime}(y)=\left(\psi(y)^{\frac{1}{n-1}}-1\right) \theta(y)^{\frac{n-2}{n-1}}-\theta(y),
\end{array}\right.
$$

and satisfy that $\phi\left(x_{0}\right)=x_{0}, \psi(r)=1, \psi^{\prime}(r-)=\frac{K+1}{r}, \psi^{\prime \prime}(r-)=\frac{K(K+1)}{r^{2}}$ and $\theta\left(x_{0}\right)=\psi\left(x_{0}\right)$.

i) Then

$$
\theta(y)=\psi(y)-\frac{K}{K+1} \frac{\psi^{\prime}(y)^{2}}{\psi^{\prime \prime}(y)} .
$$

ii) Moreover, there exists a symmetric, atom-free Nash equilibrium for the problem for which $X_{\tau^{i}}^{i}$ and $M_{\tau^{i}}^{i}$ have joint law $\nu^{*}$ that is determined by the marginal distribution $G^{*}$ of $X_{\tau^{i}}^{i}$, given by $G^{*}(x)=0$ for $x \leq 0, G^{*}(x)=1$ for $x \geq r$ and

$$
G^{*}(x)= \begin{cases}\theta\left(\phi^{-1}(x)\right)^{\frac{1}{n-1}} & , \text { if } 0<x<x_{0}, \\ \psi(x)^{\frac{1}{n-1}} & , \text { if } x_{0} \leq x<r,\end{cases}
$$

otherwise, and the conditional distribution of $M_{\tau^{i}}^{i}$ given $X_{\tau^{i}}^{i}$ such that

$$
M_{\tau^{i}}^{i}= \begin{cases}X_{\tau^{i}}^{i} & , \text { if } X_{\tau^{i}}^{i} \geq x_{0}, \\ \phi^{-1}\left(X_{\tau^{i}}^{i}\right) & , \text { if } 0 \leq X_{\tau^{i}}^{i}<x_{0} .\end{cases}
$$

Proof. The conditions in the theorem imply some properties of function $\phi$ : let $y=r-$ in (10) then since $\psi^{\prime}(r-)=\frac{K+1}{r}$ and $\psi^{\prime \prime}(r-)=\frac{K(K+1)}{r^{2}}$ we have 
$\phi(r)=0$; since (10) holds and by the positivity of $\psi^{\prime}$ and $\psi^{\prime \prime}$ we have $\phi(y)<y$

on $\left(x_{0}, r\right)$.

i) Integrating (9) with respect to $y$,

$(K+1) \theta(z)-(K+1) \theta\left(x_{0}\right)=\int_{x_{0}}^{z} \phi^{\prime}(y) \psi^{\prime}(y) d y=\phi(z) \psi^{\prime}(z)-\phi\left(x_{0}\right) \psi^{\prime}\left(x_{0}+\right)-\int_{x_{0}}^{z} \phi(y) \psi^{\prime \prime}(y) d y$.

Rearranging (10) and integrating,

$\int_{x_{0}}^{z} \phi(y) \psi^{\prime \prime}(y) d y=\int_{x_{0}}^{z} y \psi^{\prime \prime}(y) d y-\int_{x_{0}}^{z} K \psi^{\prime}(y) d y=z \psi^{\prime}(z)-x_{0} \psi^{\prime}\left(x_{0}+\right)-(1+K) \psi(z)+(1+K) \psi\left(x_{0}\right)$.

Combining this equation with (14) we find

$$
\theta(y)=\frac{1}{K+1}(\phi(y)-y) \psi^{\prime}(y)+\psi(y) .
$$

Then substituting (10) into (15), (12) follows.

ii) Let $\mathcal{E}\left(x_{0}\right)$ be the set of measures $\nu(d x, d y)$ on $[0, \infty) \times[0, \infty)$ such that $\nu(d x, d y)$ has no mass on $\left\{(x, y): y<x\right.$ or $\left.y<x_{0}\right\}$, and let $\mathcal{E}_{D}\left(x_{0}\right)$ be the subset of $\mathcal{E}\left(x_{0}\right)$ corresponding to probability measures of a pair of random variables $X \leq Y$ such that $X$ is a continuous random variable with mean $x_{0}$ and $\mathbb{E}[X-$ $z ; Y \geq z]=0$ for all $z \geq x_{0}$. Note that the last equation comes from the Doob's submartingale inequality, applied in the continuous martingale case.

Suppose that the other players all choose $F(x)$ as the target distribution of $X_{\tau}$. Then the aim of player $i$ is to choose $\nu \in \mathcal{E}\left(x_{0}\right)$ to solve

$$
\max _{\nu \in \mathcal{E}\left(x_{0}\right)}\left\{\int_{0}^{\infty} \int_{0}^{\infty}\left[(1+K) F(x)^{n-1}-K F(y)^{n-1}\right] \nu(d x, d y)\right\}
$$

subject to $\int_{0}^{\infty} \int_{0}^{\infty} x \nu(d x, d y)=x_{0}, \int_{0}^{\infty} \int_{0}^{\infty} \nu(d x, d y)=1$ and $\int_{x=0}^{\infty} \int_{y=z}^{\infty}(x-$ $z) \nu(d x, d y)=0 \forall z \geq x_{0}$.

Introduce multipliers $\lambda$ and $\gamma$ for the first two constraints, and for each $z \geq$ $x_{0}$ introduce a Lagrange multiplier $\eta(z)$ for the last constraint: the constraint becomes $\int_{0}^{\infty} \int_{0}^{\infty} \int_{z=x_{0}}^{y}\{\eta(z)(x-z) d z\} \nu(d x, d y)=0$. Then the Lagrangian for the optimization problem (16) is

$\mathcal{L}_{F}(\nu ; \lambda, \gamma, \eta)$

$$
=\int_{0}^{\infty} \int_{0}^{\infty}\left[(1+K) F(x)^{n-1}-K F(y)^{n-1}-\lambda x-\gamma-\int_{x_{0}}^{y} \eta(z)(x-z) d z\right] \nu(d x, d y)+\lambda x_{0}+\gamma .
$$

By a simple extension of the Lagrangian sufficiency theorem given in Proposition 2.1 we have that

Proposition 5.1. If $\nu^{*}, \lambda^{*}, \gamma^{*}$ and $\eta^{*}$ exist such that $\nu^{*} \in \mathcal{E}_{D}\left(x_{0}\right)$ and

$$
\mathcal{L}_{G^{*}}\left(\nu^{*} ; \lambda^{*}, \gamma^{*}, \eta^{*}\right) \geq \mathcal{L}_{G^{*}}\left(\nu ; \lambda^{*}, \gamma^{*}, \eta^{*}\right)
$$

for all $\nu \in \mathcal{E}\left(x_{0}\right)$, where $G^{*}(x)=\nu^{*}([0, x] \times(0, \infty))$, then $\nu^{*}$ is a symmetric, atom-free Nash equilibrium. 
On $[0, \infty) \times[0, \infty)$ let $\nu^{*}$ be the joint law given in the theorem and $G^{*}$ be its marginal distribution with respect to $X_{\tau}$. In particular, $G^{*}$ is given by (13). Let $\lambda^{*}=\psi^{\prime}\left(x_{0}+\right), \gamma^{*}=\psi\left(x_{0}\right)-x_{0} \psi^{\prime}\left(x_{0}+\right), \eta^{*}(y)=\psi^{\prime \prime}(y)$ for $x_{0}<y<r$, and $\eta^{*}(y)=0$ for $y \geq r$. We will show that for these multipliers (18) holds and that $\nu^{*} \in \mathcal{E}_{D}\left(x_{0}\right)$.

To prove $\nu^{*} \in \mathcal{E}_{D}\left(x_{0}\right)$, we need to show $G^{*}(0)=0, \lim _{y \uparrow \infty} G^{*}(y)=1$, $G^{*}(y)$ is continuous and non-decreasing, $\int_{0}^{\infty} u G^{*}(d u)=x_{0}$ and $\int_{x=0}^{\infty} \int_{y=z}^{\infty}(x-$ $z) \nu^{*}(d x, d y)=0$ for all $z \geq x_{0}$.

Letting $y=r-$ in (12), we find $\theta(r)=0$. Then since $\phi(r)=0$, we have $G^{*}(0)=\theta\left(\phi^{-1}(0)\right)^{1 /(n-1)}=\theta(r)^{1 /(n-1)}=0$. Moreover, $\lim _{y \uparrow \infty} G^{*}(y)=1$ follows from the finiteness of $r$. We have $\phi^{-1}\left(x_{0}\right)=x_{0}$ and hence $G^{*}$ is continuous at $x_{0}$. Since both $\phi$ and $\theta$ are decreasing and continuous on $\left[x_{0}, r\right]$, $G^{*}(x)=\theta\left(\phi^{-1}(x)\right)^{1 /(n-1)}$ is increasing for $x \in\left[0, x_{0}\right]$. Then, since $\psi(y)$ is increasing on $\left[x_{0}, r\right], G^{*}$ is continuous and non-decreasing on the whole interval of $[0, r]$. Note that this implies $r=\sup \left\{x \geq 0: G^{*}(x)<1\right\}$.

For $y>x_{0}$ we have $G^{*}(\phi(y))=\theta(y)^{1 /(n-1)}$ and so $\phi^{\prime}(y)\left(G^{*}\right)^{\prime}(\phi(y))=$ $\theta(y)^{1 /(n-1)-1} \theta^{\prime}(y) /(n-1)$. Then, using (11)

$\phi^{\prime}(y)(y-\phi(y))\left(G^{*}\right)^{\prime}(\phi(y))=\theta(y)^{\frac{2-n}{n-1}} \frac{y-\phi(y)}{n-1} \theta^{\prime}(y)=\psi(y)^{\frac{1}{n-1}}-1-\theta(y)^{\frac{1}{n-1}}=G^{*}(y)-1-G^{*}(\phi(y))$.

Hence

$\phi^{\prime}(y)(y-\phi(y))\left(G^{*}\right)^{\prime}(\phi(y))+\left(1-\phi^{\prime}(y)\right) G^{*}(\phi(y))=G^{*}(y)-1-\phi^{\prime}(y) G^{*}(\phi(y))$

and integrating from $x$ to $r$

$$
-(x-\phi(x)) G^{*}(\phi(x))=-(r-x)+\int_{x}^{r} G^{*}(y) d y+\int_{0}^{\phi(x)} G^{*}(y) d y .
$$

Then, setting $x=x_{0}$ we recover $x_{0}=\int_{0}^{r}\left(1-G^{*}(y)\right) d y$ so that a random variable with distribution function $G^{*}$ has mean $x_{0}$.

Finally, from its construction we have that $\nu^{*}$ only puts mass on $A$. Hence, from (8),

$$
\int_{x=0}^{\infty} \int_{y=z}^{\infty}(x-z) \nu^{*}(d x, d y)=\int_{0}^{\phi(z)}(x-z) G^{*}(d x)+\int_{z}^{r}(x-z) G^{*}(d x)=0 .
$$

Now we prove that (18) holds. Let $L^{*}(x, y)=(1+K) G^{*}(x)^{n-1}-K G^{*}(y)^{n-1}-$ $\lambda^{*} x-\gamma^{*}-\int_{x_{0}}^{y} \eta^{*}(z)(x-z) d z$ and then $\mathcal{L}_{G^{*}}\left(\nu ; \lambda^{*}, \gamma^{*}, \eta^{*}\right)=\int_{0}^{\infty} \int_{0}^{\infty} L^{*}(x, y) \nu(d x, d y)+$ $\lambda^{*} x_{0}+\gamma^{*}$.

For notational convenience, extend the domain of $\psi$ to $[0, r]$ by defining $\psi(x)=\theta\left(\phi^{-1}(x)\right)$ for $x \in\left[0, x_{0}\right)$. Then, for $x<x_{0}, \psi^{\prime}(x)=\frac{\theta^{\prime}\left(\phi^{-1}(x)\right)}{\phi^{\prime}\left(\phi^{-1}(x)\right)}=$ $\frac{\psi^{\prime}\left(\phi^{-1}(x)\right)}{K+1}>0$ where the last equality comes from (9). Moreover $\psi^{\prime \prime}(x)=$ $\frac{\psi^{\prime \prime}\left(\phi^{-1}(x)\right)}{(1+K) \phi^{\prime}\left(\phi^{-1}(x)\right)}<0$. Thus $\psi$ is increasing on $[0, r], \psi^{\prime \prime}(x)<0$ if $x \in\left(0, x_{0}\right)$ and $\psi^{\prime \prime}(x)>0$ if $x \in\left(x_{0}, r\right)$. 
Fix $y \in\left[x_{0}, r\right]$. For any $0 \leq x \leq y$,

$$
\begin{aligned}
L^{*}(x, y) & =(1+K) \psi(x)-K \psi(y)-\psi^{\prime}\left(x_{0}+\right) x-\psi\left(x_{0}\right)+x_{0} \psi^{\prime}\left(x_{0}+\right)-\int_{x_{0}}^{y} \psi^{\prime \prime}(z)(x-z) d z \\
& =(1+K)(\psi(x)-\psi(y))+(y-x) \psi^{\prime}(y) .
\end{aligned}
$$

We have $L^{*}(\phi(y), y)=(1+K)(\theta(y)-\psi(y))+(y-\phi(y)) \psi^{\prime}(y)$ and then by (12) and (10), $L^{*}(\phi(y), y)=0$. It is also clear that $L^{*}(y, y)=0$.

Differentiating (201) with respect to $x$,

$$
\frac{\partial L^{*}}{\partial x}(x, y)=(1+K) \psi^{\prime}(x)-\psi^{\prime}(y) ; \quad \frac{\partial^{2} L^{*}}{\partial x^{2}}(x, y)=(1+K) \psi^{\prime \prime}(x) .
$$

Then $\frac{\partial^{2} L^{*}}{\partial x^{2}}(x, y)<0$ on $\left(0, x_{0}\right)$ and $\frac{\partial^{2} L^{*}}{\partial x^{2}}(x, y)>0$ on $\left(x_{0}, y\right)$. Since $\frac{\partial L^{*}}{\partial x}(\phi(y), y)=$ $(1+K) \psi^{\prime}(\phi(y))-\psi^{\prime}(y)=(1+K) \frac{\psi^{\prime}(y)}{K+1}-\psi^{\prime}(y)=0$ and $\frac{\partial L^{*}}{\partial x}(y, y)=K \psi^{\prime}(y)>0$, it follows that $\frac{\partial L^{*}}{\partial x}(x, y)>0$ if $x \in(0, \phi(y)), \frac{\partial L^{*}}{\partial x}(x, y)<0$ if $x \in(\phi(y), \tilde{x})$ and $\frac{\partial L^{*}}{\partial x}(x, y)>0$ if $x \in(\tilde{x}, y)$, where $\tilde{x} \in\left(x_{0}, y\right)$ is such that $\left.\frac{\partial L^{*}}{\partial x}(x, y)\right|_{x=\tilde{x}}=0$. It follows that $L^{*}(x, y)<0$ for $x \in[0, \phi(y)) \cup(\phi(y), y)$.

Now fix $y>r$. For any $0 \leq x \leq y$, and writing $\tilde{\psi}(x)=\psi(x)-x / r$,

$$
\begin{aligned}
L^{*}(x, y) & =(1+K) \psi(x)-K-\psi^{\prime}\left(x_{0}+\right) x-\psi\left(x_{0}\right)+x_{0} \psi^{\prime}\left(x_{0}+\right)-\int_{x_{0}}^{r} \psi^{\prime \prime}(z)(x-z) d z \\
& =(1+K)(\psi(x)-1)+(r-x) \psi^{\prime}(r-)=(1+K)\left(\psi(x)-\frac{x}{r}\right)=(1+K) \tilde{\psi}(x) .
\end{aligned}
$$

If $x \in(r, y]$ then $L^{*}(x, y)=(1+K) \frac{1}{r}[r-x]<0$. Now suppose $x \in(0, r)$. Since $\phi(r)=0$, we have $\psi^{\prime}(0+)=\frac{1}{r}$ and thus $\tilde{\psi}^{\prime}(0+)=0$. Further $\tilde{\psi}^{\prime}(r-)=\frac{K}{r}>0$. Then by the sign of $\psi^{\prime \prime}(x)$, we get $\tilde{\psi}^{\prime}(x)$ is negative and then positive on $(0, r)$. Since $\tilde{\psi}(0)=\tilde{\psi}(r)=0$, we deduce that $\tilde{\psi}(x)<0$ on $(0, r)$. Thus $L^{*}(x, y)<0$ for $x \in(0, r)$.

From above analysis, we know $L^{*}(x, y) \leq 0$ for any $(x, y)$ such that $0 \leq x \leq y$ and $y \geq x_{0}$. This means that $\forall \nu \in \mathcal{E}\left(x_{0}\right)$

$$
\begin{aligned}
\mathcal{L}_{G^{*}}\left(\nu ; \lambda^{*}, \gamma^{*}, \eta^{*}\right) & =\int_{0}^{\infty} \int_{0}^{\infty} L^{*}(x, y) \nu(d x, d y)+\lambda^{*} x_{0}+\gamma^{*} \\
& =\int_{0}^{\infty} \int_{0}^{\infty} L^{*}(x, y) \nu(d x, d y)+\psi\left(x_{0}\right) \leq \psi\left(x_{0}\right)=\mathcal{L}_{G^{*}}\left(\nu^{*} ; \lambda^{*}, \gamma^{*}, \eta^{*}\right) .
\end{aligned}
$$

Thus $\nu^{*}$ is a symmetric, atom-free Nash equilibrium from Proposition 5.1

It remains to show that there exists a constant $r$ and functions $(\phi, \theta, \psi)$ which satisfy the hypotheses of the Theorem 5.1 and hence that a symmetric atomfree Nash equilibrium always exists. The following lemma is key in defining the appropriate entities.

Lemma 5.1. Let $J(u)$ solve the ordinary differential equation

$$
J^{\prime}(u)=\frac{J(u)+1-(1-u)^{1 /(n-1)}}{(K+1)\left[1-u-J(u)^{n-1}\right]}
$$


subject to $J(0)=0$ and $u \geq 0$. Let $u^{*}=\sup \left\{u: J(u)<(1-u)^{1 /(n-1)}\right\}$.

i) Define

$$
H(z)=\frac{K}{(K+1)\left[z-J(1-z)^{n-1}\right]}
$$

on $\left[z^{*}, 1\right]$, where $z^{*}=1-u^{*}$. Then $z^{*}>0, H$ is positive on $\left(z^{*}, 1\right)$ and $\int_{z^{*}}^{1} \exp \left(\int_{w}^{1} H(v) d v\right) d w<(K+1)$.

ii) Define

$$
r=\frac{x_{0}(K+1)}{(K+1)-\int_{z^{*}}^{1} \exp \left(\int_{w}^{1} H(v) d v\right) d w}
$$

and

$$
\Psi(z)=\frac{r}{K+1}\left[(K+1)-\int_{z}^{1} \exp \left(\int_{w}^{1} H(v) d v\right) d w\right]
$$

on $\left[z^{*}, 1\right]$. Let $\psi=\Psi^{-1}$ be the inverse function of $\Psi$. Then $x_{0}<r<\infty$ and $\psi:\left[x_{0}, r\right] \mapsto[0,1]$ is a strictly increasing and strictly convex function that satisfies $\psi(r)=1, \psi^{\prime}(r-)=\frac{K+1}{r}$ and $\psi^{\prime \prime}(r-)=\frac{K(K+1)}{r^{2}}$.

iii) Define

$$
\phi(y)=y-\frac{K \psi^{\prime}(y)}{\psi^{\prime \prime}(y)} .
$$

Then $\phi:\left[x_{0}, r\right] \mapsto\left[0, x_{0}\right]$ is a strictly decreasing function with $\phi\left(x_{0}\right)=x_{0}$.

iv) Define

$$
\theta(y)=\psi\left(x_{0}\right)+\frac{1}{K+1} \int_{x_{0}}^{y} \phi^{\prime}(z) \psi^{\prime}(z) d z
$$

Then $\theta:\left[x_{0}, r\right] \mapsto[0,1]$ is a strictly decreasing function with $\theta\left(x_{0}\right)=\psi\left(x_{0}\right)$. Moreover, $\theta(y)=\psi(y)-(y-\phi(y)) \psi^{\prime}(y) /(K+1)$.

Proof. It is easily seen that $J(u)$ is a strictly increasing function at least until $J(u)=\sqrt[n-1]{1-u}$, and that $u^{*}<1$.

i) Since $u^{*}<1, z^{*}=1-u^{*}>0$. Since $J$ is increasing, for any $u \in\left(0, u^{*}\right)$ $J(u)^{n-1} \leq J\left(u^{*}\right)^{n-1}$ and thus $1-u-J(u)^{n-1} \geq 1-u-J\left(u^{*}\right)^{n-1}=u^{*}-u$. This means for any $z \in\left(z^{*}, 1\right)$ we have $z-J(1-z)^{n-1} \geq z-z^{*}>0$ and then $0<H(z) \leq \frac{K}{(K+1)\left(z-z^{*}\right)}$. Moreover,

$$
\begin{aligned}
\int_{z^{*}}^{1} \exp \left(\int_{w}^{1} \frac{K}{(K+1)\left(v-z^{*}\right)} d v\right) d w & =\int_{z^{*}}^{1} \exp \left(\frac{K}{K+1} \ln \frac{1-z^{*}}{w-z^{*}}\right) d w=\int_{z^{*}}^{1}\left(\frac{1-z^{*}}{w-z^{*}}\right)^{\frac{K}{K+1}} d w \\
& =\left(1-z^{*}\right)^{\frac{K}{K+1}}\left(1-z^{*}\right)^{\frac{1}{K+1}}(K+1)=\left(1-z^{*}\right)(K+1)<(K+1),
\end{aligned}
$$

and it follows that $\int_{z^{*}}^{1} \exp \left(\int_{w}^{1} H(v) d v\right) d w<(K+1)$.

ii) Since $0<(K+1)-\int_{z^{*}}^{1} \exp \left(\int_{w}^{1} H(v) d v\right) d w<(K+1)$, we have that $x_{0}<$ $r<\infty$. Taking derivatives of $\Psi$ on $\left(z^{*}, 1\right)$, we find $\Psi^{\prime}(z)=\frac{r}{K+1} \exp \left(\int_{z}^{1} H(v) d v\right)>$ 0 and $\Psi^{\prime \prime}(z)=-H(z) \Psi^{\prime}(z)<0$. Then since $\Psi\left(z^{*}\right)=x_{0}$ and $\Psi(1)=r, \Psi$ is 
a strictly increasing and strictly concave function from $\left[z^{*}, 1\right]$ to $\left[x_{0}, r\right]$. Thus $\psi=\Psi^{-1}:\left[x_{0}, r\right] \mapsto[0,1]$ is a strictly increasing and strictly convex function satisfying $\psi(r)=1$.

Moreover, $\psi^{\prime}(y)=\frac{1}{\Psi^{\prime}(\psi(y))}$ and thus $\psi^{\prime \prime}(y)=-\frac{\Psi^{\prime \prime}(\psi(y)) \psi^{\prime}(y)}{\Psi^{\prime}(\psi(y))^{2}}$. Then since $\Psi(1)=r, \Psi^{\prime}(1-)=\frac{r}{K+1}$ and $\Psi^{\prime \prime}(1-)=-\frac{r K}{(K+1)^{2}}$, we get $\psi(r)=1, \psi^{\prime}(r-)=$ $\frac{1}{\Psi^{\prime}(1-)}=\frac{K+1}{r}$ and $\psi^{\prime \prime}(r-)=-\frac{\Psi^{\prime \prime}(1-) \psi^{\prime}(r-)}{\Psi^{\prime}(1-)^{2}}=\frac{K(K+1)}{r^{2}}$.

iii) Letting $u=1-z$ in (21), we get $J^{\prime}(1-z)=\frac{J(1-z)+1-z^{1 /(n-1)}}{(K+1)\left[z-J(1-z)^{n-1}\right]}=$ $\frac{H(z)}{K}\left(J(1-z)+1-z^{\frac{1}{n-1}}\right)$. Thus,

$$
\begin{aligned}
H^{\prime}(z) & =-\frac{K\left[1+(n-1) J(1-z)^{n-2} J^{\prime}(1-z)\right]}{(K+1)\left[z-J(1-z)^{n-1}\right]^{2}} \\
& =-\frac{K+1}{K} H(z)^{2}\left[1+(n-1) J(1-z)^{n-2} \frac{H(z)}{K}\left(J(1-z)+1-z^{\frac{1}{n-1}}\right)\right] \\
& =-\frac{K+1}{K} H(z)^{2}-\frac{(K+1)(n-1)}{K^{2}} H(z)^{3}\left[J(1-z)^{n-1}+J(1-z)^{n-2}\left(1-z^{\frac{1}{n-1}}\right)\right]
\end{aligned}
$$

It is clear from the middle line above that $\frac{H^{\prime}(z)}{H(z)^{2}}<-\frac{K+1}{K}$.

Note that $\phi^{\prime}(y)=1-K\left(1-\frac{\psi^{\prime}(y) \psi^{\prime \prime \prime}(y)}{\psi^{\prime \prime}(y)^{2}}\right)$. Since $H(z)=-\frac{\Psi^{\prime \prime}(z)}{\Psi^{\prime}(z)}=\frac{\psi^{\prime \prime}(\Psi(z))}{\psi^{\prime}(\Psi(z))^{2}}$, we have that

$$
H(\psi(y))=\frac{\psi^{\prime \prime}(y)}{\psi^{\prime}(y)^{2}}
$$

and thus $H^{\prime}(\psi(y)) \psi^{\prime}(y)=\frac{\psi^{\prime \prime \prime}(y)}{\psi^{\prime}(y)^{2}}-\frac{2 \psi^{\prime \prime}(y)^{2}}{\psi^{\prime}(y)^{3}}$. Thus $1-\frac{\psi^{\prime}(y) \psi^{\prime \prime \prime}(y)}{\psi^{\prime \prime}(y)^{2}}=-H^{\prime}(\psi(y)) \frac{\psi^{\prime}(y)^{4}}{\psi^{\prime \prime}(y)^{2}}-$ 1 and therefore

$$
\phi^{\prime}(y)=K \frac{H^{\prime}(\psi(y))}{H(\psi(y))^{2}}+K+1 \text {. }
$$

It follows that $\phi^{\prime}(y)<0$.

Since $\psi^{\prime}(r-)=\frac{K+1}{r}, \psi^{\prime}(y)>0$ and $\psi^{\prime \prime}(y)>0$ on $\left(x_{0}, r\right)$, we know $\psi^{\prime}\left(x_{0}+\right)$ is bounded. Then, since $\frac{\psi^{\prime \prime}\left(x_{0}+\right)}{\psi^{\prime}\left(x_{0}+\right)^{2}}=H\left(\psi\left(x_{0}\right)\right)=H\left(z^{*}\right)=+\infty$, we get $\psi^{\prime \prime}\left(x_{0}+\right)=$ $+\infty$. Substituting these values into (22) we obtain $\phi\left(x_{0}\right)=x_{0}$.

iv) The statements about $\theta$ are either trivial, or follow as in the derivation of $(15)$.

Theorem 5.2. Let $r, \psi, \phi, \theta$ be as defined in Lemma 5.1.

Then there exists a symmetric, atom-free Nash equilibrium for the problem for which $X_{\tau^{i}}^{i}$ has distribution $F$ where $F(x)=0$ for $x \leq 0, F(x)=1$ for $x \geq r$ and otherwise

$$
F(x)= \begin{cases}\theta\left(\phi^{-1}(x)\right)^{\frac{1}{n-1}} & \text { if } 0<x<x_{0} \\ \psi(x)^{\frac{1}{n-1}} & \text { if } x_{0} \leq x<r .\end{cases}
$$


Proof. By (22) and (24), $\frac{1}{H(\psi(y))}=\frac{\psi^{\prime}(y)^{2}}{\psi^{\prime \prime}(y)}=\frac{y-\phi(y)}{K} \psi^{\prime}(y)$. Hence $\theta(y)=\psi(y)-$ $\frac{K}{(K+1) H(\psi(y))}=J(1-\psi(y))^{n-1}$. Thus letting $z=\psi(y)$ in (23),

$H^{\prime}(\psi(y))=-\frac{K+1}{K} H(\psi(y))^{2}-\frac{(K+1)(n-1)}{K^{2}} H(\psi(y))^{3}\left[\theta(y)+\left(1-\psi(y)^{\frac{1}{n-1}}\right) \theta(y)^{\frac{n-2}{n-1}}\right]$,

and using (25) and rearranging above equation,

$$
\frac{y-\phi(y)}{n-1} \frac{\phi^{\prime}(y) \psi^{\prime}(y)}{K+1}=-\left[\theta(y)+\left(1-\psi(y)^{\frac{1}{(n-1)}}\right) \theta(y)^{\frac{n-2}{n-1}}\right] .
$$

Then substituting (9) into above equation, (11) follows. Therefore, (†) holds. Then using Theorem 5.1 we obtain the symmetric, atom-free Nash equilibrium given in Theorem 5.2

Example 5.1. As an example we consider a 3-player contest. Set $x_{0}=1$ and $n=3$. In Figures ?? and ?? we give graphs of the optimal distribution $G^{*}(x)$ and its density function $g^{*}(x)$ for various values of $K$.

Figure 1 here.

Figure 2 here

As we can seen in Figure ??, the right endpoint $r=r(K)$ of $G^{*}(x)$ decreases as $K$ increases. Moreover $r(K)$ tends to $n=3$ as $K$ decreases to 0 and tends to $x_{0}$ as $K$ increases to $+\infty$. We also find that $G^{*}(x)$ tends to the equilibrium distribution of the original contest as $K$ decreases to 0 and $G^{*}(x)$ tends to the Heaviside function $\mathcal{H}_{\left\{x \geq x_{0}\right\}}$ as $K$ increases to $+\infty$. From Figure ??, we find $g^{*}(x)$ jumps at $x_{0}$ if $K>0$ and $g^{*}(x)$ tends to $+\infty$ as $y$ tends to 0 .

Intuitively, if $K$ is very large then the player does not aim for large values of the stopped process, for then she risks a moderate value of the maximum together with a small and losing value for the stopped process. Because of the large penalty she wishes to avoid such outcomes.

Example 5.2. In the 2-player contest we can give explicit expressions for several of the quantities of interest.

Set $n=2$. Substituting (9), (10) and (12) into (11), we get

$$
(y-\phi(y)) \frac{1}{1+K} \phi^{\prime}(y) \psi^{\prime}(y)=(\psi(y)-1)-\psi(y)+\frac{K}{K+1} \frac{y-\phi(y)}{K} \psi^{\prime}(y) .
$$

Defining $\varphi(y)=y-\phi(y)$ the above equation simplifies to $\varphi(y) \varphi^{\prime}(y)=\frac{K+1}{\psi^{\prime}(y)}$. Differentiating this expression and using (10) we have

$$
\left[\varphi(y) \varphi^{\prime}(y)\right]^{\prime}=-(K+1) \frac{\psi^{\prime \prime}(y)}{\psi^{\prime}(y)^{2}}=-(K+1) \frac{K}{\varphi(y) \psi^{\prime}(y)}=-K \varphi^{\prime}(y),
$$

and then

$$
\varphi(y) \varphi^{\prime}(y)=-K \varphi(y)+K \varphi(r)+\varphi(r) \varphi^{\prime}(r-)
$$


Since $\varphi(r)=r$ and $\psi^{\prime}(r-)=\frac{K+1}{r}$ we have $\varphi^{\prime}(r-)=1$. Then (26) becomes $\varphi(y) \varphi^{\prime \prime}(y)=-K \varphi(y)+r(K+1)$, which using the boundary condition $\varphi(r)=r$ has solution

$$
r-y=\frac{r(K+1)}{K^{2}} \ln \left((K+1)-\frac{K \varphi(y)}{r}\right)-\frac{r-\varphi(y)}{K}
$$

Using $\varphi\left(x_{0}\right)=x_{0}-\phi\left(x_{0}\right)=0$, we find

$$
r=x_{0} \frac{K^{2}}{(K+1)[K-\ln (1+K)]}
$$

and therefore the implicit form of $\varphi(y)$ for $y \in\left[x_{0}, r\right]$ is

$$
y=x_{0}-\frac{\varphi(y)}{K}-\frac{x_{0}}{K-\ln (1+K)} \ln \left[1-\varphi(y) \frac{K-\ln (1+K)}{K x_{0}}\right]
$$

and $\phi(y)=y-\varphi(y)$. It is possible to express $\psi$ and $\theta$ in terms of $\varphi$, and thence the optimal distribution $G^{*}$ of $X_{\tau^{i}}^{i}$ and the optimal conditional distribution of $M_{\tau^{i}}^{i}$ given $X_{\tau^{i}}^{i}$, but these expressions are not so compact.

\section{Contest with regret over failure to stop at the best time}

In this section we discuss the contest with regret over failure to stop at the best time. A player experiences regret if she could have won if she had stopped at the maximum value over the whole path. The maximum value $M^{i}$ is given by

$$
M^{i}:=M_{H_{0}^{i}}^{i}=\sup _{0 \leq t \leq H_{0}^{i}} X_{t}^{i} .
$$

Theorem 6.1. There exists a symmetric, atom-free Nash equilibrium for the problem for which $X_{\tau^{i}}^{i}$ has law $F(x)$, where for $x \geq 0$

$$
F(x)=\min \left\{\sqrt[n-1]{\frac{x}{n x_{0}}}, 1\right\} .
$$

Proof. The agent's expected payoff is

$$
(1+K) \mathbb{E}\left[F\left(X_{\tau^{i}}^{i}\right)^{n-1}\right]-K \mathbb{E}\left[F\left(M_{H_{0}^{i}}^{i}\right)^{n-1}\right]
$$

But the latter term is independent of the stopping rule used by the agent. Hence, in determining her optimal strategy the agent need only consider $(1+$ $K) \mathbb{E}\left[F\left(X_{\tau^{i}}^{i}\right)^{n-1}\right]$. Modulo the initial constant, this is the same objective function as in the standard case.

Remark 6.1. The agent follows exactly the same Nash equilibrium strategy as an agent in the original contest, in which there is no penalty. The intuition behind is that the regret is determined by $M_{H_{0}^{i}}^{i}$ but player cannot change the distribution of $M_{H_{0}^{i}}^{i}$ by changing the choices of stopping time $\tau^{i}$. 


\section{Derivation of the equilibrium distribution}

This section is intended to illustrate how we derived the optimal multipliers and the candidate Nash equilibrium in Sections 2, 4 and 5 and also the boundary conditions in Section 5. The Lagrangian approach gives a general method for finding the optimal solution, which is distinct from the ideas in Seel and Strack [6], and can be generalised to other settings.

\subsection{Contest without regret or with regret over stopping too soon}

Recall the definition of the Lagrangian $\mathcal{L}_{F}(G ; \lambda, \gamma)$ for the optimization problem (11) and (5). (We cover the more complicated case of regret from continuing beyond a winning time in a separate section.)

Denote by $L_{F}(x)$ by the integrand in $\mathcal{L}_{F}$, so that $\mathcal{L}_{F}(G ; \lambda, \gamma)=\int_{0}^{\infty} L_{F}(x) G(d x)+$ $\lambda x_{0}+\gamma$. In order to have a finite optimal solution we require $L_{F}(x) \leq 0$ on $[0, \infty)$. Let $\mathcal{D}_{F}$ be the set of $(\lambda, \gamma)$ such that $\mathcal{L}_{F}(\cdot ; \lambda, \gamma)$ has a finite maximum. Then $\mathcal{D}_{F}$ is defined by

$$
\mathcal{D}_{F}=\left\{(\lambda, \gamma): L_{F}(x) \leq 0 \text { on }[0, \infty)\right\}
$$

In order to reach the maximum value, we require $G(d x)=0$ when $L_{F}(x)<0$. This means that for $(\lambda, \gamma) \in \mathcal{D}_{F}$ the maximum of $\mathcal{L}_{F}(\cdot ; \lambda, \gamma)$ occurs at $G^{*}$ such that $G^{*}(d x)=0$ when $L_{F}(x)<0$. Conversely we expect that $G^{*}(d x)>0$ when $L_{F}(x)=0$. If the Nash equilibrium is symmetric then we must have $G^{*}(x)=F(x)$ and then $L_{G^{*}}(x) \leq 0$, and $L_{G^{*}}(x)=0$ when $G^{*}(d x)>0$. Introduce $a=\inf \left\{x: G^{*}(x)>0\right\}$ and $b=\sup \left\{x: G^{*}(x)<1\right\}$ which are the limits on the support of $G^{*}$.

\subsubsection{Contest without regret}

For the optimization problem (1), $L_{F}(x)=F(x)^{n-1}-\lambda x-\gamma$. Observe that $0 \leq F(0)^{n-1}$ so that if $(\lambda, \gamma) \in \mathcal{D}_{F}$ then $\gamma$ is non-negative.

Since $L_{G^{*}}(x)=G^{*}(x)^{n-1}-\lambda x-\gamma$, we must have $G^{*}(x)=\sqrt[n-1]{\lambda x+\gamma}$ at least when $G^{*}(d x)>0$. Since we are searching for atom-free solutions we must have $G^{*}(x)=\sqrt[n-1]{\lambda x+\gamma}$ on the whole of the interval $[a, b]$. Moreover, since $G^{*}$ is non-decreasing and not constant we must have $\lambda>0$.

Since $G^{*}$ is atom-free, $G^{*}(a)=0$ and hence $\lambda a+\gamma=0$. Then by the nonnegativity of $a$ and $\gamma$ and the positivity of $\lambda$ it follows that $\gamma=0=a$. Thus $G^{*}(x)=\sqrt[n-1]{\lambda x}$ on $[0, b]$ for some $\lambda$ and $b$ which we must find.

For a feasible solution, $\int_{0}^{\infty} G^{*}(d x)=1$ and $\int_{0}^{\infty} x G^{*}(d x)=x_{0}$, so that

$1=\int_{0}^{b} d(\sqrt[n-1]{\lambda x})=\sqrt[n-1]{\lambda b} . \quad x_{0}=\int_{0}^{b} x d(\sqrt[n-1]{\lambda x})=\frac{\sqrt[n-1]{\lambda}}{n} b^{\frac{n}{n-1}}=\sqrt[n-1]{\lambda b} \frac{b}{n}$

Hence $b=n x_{0}$ and $\lambda=1 /\left(n x_{0}\right)$. This gives us that $G^{*}$ is the distribution function given in Theorem 2.2 . 


\subsubsection{Contest with regret from stopping too soon}

Now we have that $L_{F}(x)=(1+K) F(x)^{n-1}-K x \int_{x}^{\infty} \frac{F(y)^{n-1}}{y^{2}} d y-\lambda x-\gamma$. Then $L_{F}(0)=(1+K) F(0)^{n-1}-\gamma$ and as before, if $(\lambda, \gamma) \in \mathcal{D}_{F}$ then $\gamma$ is non-negative.

Let $\psi(x)=G^{*}(x)^{n-1}$ then $L_{G^{*}}(x)$ becomes

$$
L_{G^{*}}(x)=(1+K) \psi(x)-K x \int_{x}^{\infty} \frac{\psi(y)}{y^{2}} d y-\lambda x-\gamma .
$$

Thus we expect $\psi(x)$ is the solution to $L_{G^{*}}(x)=0$ at least when $\psi(d x)>0$.

Setting $L_{G^{*}}(x)=0$ and differentiating (27) twice with respect to $x$, we find

$$
(1+K) \psi^{\prime \prime}(x) x+K \psi^{\prime}(x)=0 .
$$

Thus $\psi(x)=C_{1} x^{\frac{1}{K+1}}+C_{2}$, where $C_{1}$ and $C_{2}$ are some constants, and then $G^{*}(x)=\sqrt[n-1]{C_{1} x^{\frac{1}{K+1}}+C_{2}}$ at least when $G^{*}(d x)>0$. Since we are seeking an atom-free solution we must have $G^{*}(x)=\sqrt[n-1]{C_{1} x^{\frac{1}{k+1}}+C_{2}}$ on the whole interval of $[a, b]$, where $C_{1}>0$.

Substituting $\psi(x)=G^{*}(x)^{n-1}=\left(C_{1} x^{\frac{1}{K+1}}+C_{2}\right) \wedge 1$ into (27), and setting $L_{G^{*}}(x)=0$ we have $\forall x \in[a, b]$

$$
\begin{aligned}
0 & =(1+K)\left(C_{1} x^{\frac{1}{K+1}}+C_{2}\right)-K x \int_{x}^{b} \frac{C_{1} y^{\frac{1}{K+1}}+C_{2}}{y^{2}} d y-K x \int_{b}^{\infty} \frac{1}{y^{2}} d y-\lambda x-\gamma \\
& =\left[(1+K) C_{1} b^{\frac{1}{K+1}}+K C_{2}-K-\lambda b\right] \frac{x}{b}+C_{2}-\gamma
\end{aligned}
$$

This gives us optimal multipliers $\gamma^{*}=C_{2}$ and $\lambda^{*}=\frac{1}{b}\left[(1+K) C_{1} b^{\frac{1}{K+1}}+K C_{2}-K\right]$.

Since $G^{*}$ is atom-free, $G^{*}(a)=0$ and hence $C_{1} a^{\frac{1}{K+1}}+C_{2}=0$, and from the non-negativity of $a$ and $\gamma^{*}=C_{2}$ and the positivity of $C_{1}$ it follows that $C_{2}=a=0$. Thus $G^{*}(x)=\sqrt[n-1]{C_{1} x^{\frac{1}{K+1}}}$ on $[0, b]$ for some $C_{1}$ and $b$ which can be identified using the fact that $G^{*}$ corresponds to a probability distribution with mean $x_{0}$. In particular, setting $N=1+(K+1)(n-1)$ for a feasible solution,

$$
\left\{\begin{array}{l}
1=\int_{0}^{b} d\left(\sqrt[n-1]{C_{1} x^{\frac{1}{K+1}}}\right)=\sqrt[n-1]{C_{1} b^{1 /(K+1)}} \\
x_{0}=\int_{0}^{b} x d\left(\sqrt[n-1]{C_{1} x^{\frac{1}{K+1}}}\right)=\frac{n-\sqrt[1]{C_{1}}}{(K+1)(n-1)+1} b^{\frac{(K+1)(n-1)+1}{(K+1)(n-1)}}=\sqrt[N-1]{C_{1}^{K+1}} b \frac{b}{N}
\end{array}\right.
$$

Hence $C_{1}=b^{-1 /(K+1)}$ and then $b=N x_{0}$ and $C_{1}=\sqrt[K+1]{\frac{1}{N x_{0}}}$. Thus $G^{*}(x)=$ $\sqrt[N-1]{x / N x_{0}}$ on $\left[0, N x_{0}\right]$. 


\subsection{Contest with regret over past failure}

Recall the definition of the Lagrangian $\mathcal{L}_{F}(\nu ; \lambda, \gamma, \eta)$ for the optimization problem of Section 5. Let $L_{F}(x, y)$ be the integrand in the definition of $\mathcal{L}_{F}$ as given in (17). In order to have a finite optimal solution we require $L_{F}(x, y) \leq 0$ on $[0, \infty) \times\left[x_{0}, \infty\right)$. Let $\mathcal{D}_{F}$ be the set of $(\lambda, \gamma, \eta)$ such that $\mathcal{L}_{F}(\cdot ; \lambda, \gamma, \eta)$ has a finite maximum. Then $\mathcal{D}_{F}$ is defined by

$$
\mathcal{D}_{F}=\left\{(\lambda, \gamma, \eta): L_{F}(x, y) \leq 0 ; x \geq 0, y \geq x_{0}\right\} .
$$

For $(\lambda, \gamma, \eta) \in \mathcal{D}_{F}$ the maximum of $\mathcal{L}_{F}(\cdot ; \lambda, \gamma, \eta)$ occurs at a measure $\nu^{*}$ such that $\nu^{*}(d x, d y)=0$ when $L_{F}(x, y)<0$. Conversely we expect that $\nu^{*}(d x, d y)>$ 0 when $L_{F}(x, y)=0$.

Let $G^{*}(x)=\nu^{*}\left(\left\{(u, y): u \leq x, x_{0} \leq y<\infty\right\}\right)$ be the marginal of $\nu^{*}$. If the Nash equilibrium is symmetric then we must have $G^{*}(x)=F(x)$ and $L_{G^{*}}(x, y)=0$ when $\nu^{*}(d x, d y)>0$. Motivated by the results of previous sections we expect $G^{*}$ to place mass on an interval $[a, b]$ where $0=a<x_{0}<b$. In this section we write $b=r$ for the upper limit.

It follows from the discussion before Theorem 5.1 that for an optimal solution either $X_{\tau^{i}}^{i}=M_{\tau^{i}}^{i}$ or $X_{\tau^{i}}^{i}=\phi\left(M_{\tau^{i}}^{i}\right)$ for some decreasing function $\phi$. Hence, for $x_{0} \leq y \leq r$ we expect $\nu^{*}(d x, d y)>0$ if and only if either $x=y$ or $x=\phi(y)$. Let $\psi(x)=G^{*}(x)^{n-1}$. Then $L_{G^{*}}(x, y)$ becomes

$$
L(x, y):=L_{G^{*}}(x, y)=(1+K) \psi(x)-K \psi(y)-\lambda x-\gamma-\int_{x_{0}}^{y} \eta(z)(x-z) d z .
$$

Fixing $y \in\left(x_{0}, r\right)$, and using $L(x, y) \leq 0$ for any $0 \leq x \leq y$, together with $L(\phi(y), y)=0$ we expect $\frac{\partial L}{\partial x}(\phi(y), y)=0$.

Thus $\forall y \in\left(x_{0}, r\right), \psi$ and $\phi$ must solve

$$
\left\{\begin{array}{l}
L(y, y)=\psi(y)-\lambda y-\gamma-\int_{x_{0}}^{y} \eta(z)(y-z) d z=0 \\
L(\phi(y), y)=(1+K) \psi(\phi(y))-K \psi(y)-\lambda \phi(y)-\gamma-\int_{x_{0}}^{y} \eta(z)(\phi(y)-z) d z \\
\frac{\partial L}{\partial x}(\phi(y), y)=(1+K) \psi^{\prime}(\phi(y))-\lambda-\int_{x_{0}}^{y} \eta(z) d z=0
\end{array}\right.
$$

Differentiating (28) with respect to $y$, yields

$$
\psi^{\prime}(y)-\lambda-\int_{x_{0}}^{y} \eta(z) d z=0 .
$$

Comparing (30) with (31), we find $\psi^{\prime}(y)=(1+K) \psi^{\prime}(\phi(y))$. If we now set $\theta(y)=\psi(\phi(y))$, then (91) follows. From (31) we find

$$
\psi^{\prime \prime}(y)-\eta(y)=0
$$


Then, differentiating (29) with respect to $y$, and using (30) we obtain $-K \psi^{\prime}(y)-$ $\eta(y)(\phi(y)-y)=0$, and (10). Finally, (11) comes directly from (8) on noting that $G(\phi(m))=\theta(m)^{1 /(n-1)}$.

Next we deduce the boundary conditions. First note that from (6) we can infer that $\phi\left(x_{0}\right)=x_{0}$ and $\phi(r)=0$. Hence $\theta\left(x_{0}\right)=\psi\left(x_{0}\right)$ and $\theta(r)=0$. Given that (9) and (10) hold, as in the proof of Theorem [5.1] we have that (15) holds. Letting $y=r$ and using $\psi(r)=1, \phi(r)=0$ and $\theta(r)=0$, we find $0=\frac{-r}{K+1} \psi^{\prime}(r-)+\psi(r)$ and hence $\psi^{\prime \prime}(r-)=\frac{K+1}{r}$. Further, letting $y=r$ in (10) we get $\psi^{\prime \prime}(r-)=K(K+1) / r^{2}$ as required.

Lastly, we derive the optimal multipliers which we write as $\eta^{*}, \lambda^{*}$ and $\gamma^{*}$. From (32), $\eta^{*}(y)=\psi^{\prime \prime}(y)$ for $y \in\left(x_{0}, r\right)$. Then, from (31), $\lambda^{*}=\psi^{\prime}(y)-$ $\int_{x_{0}}^{y} \eta^{*}(z) d z=\psi^{\prime}(y)-\int_{x_{0}}^{y} \psi^{\prime \prime}(z) d z=\psi^{\prime}\left(x_{0}+\right)$. Finally (28) yields,

$\gamma^{*}=\psi(y)-\lambda y-\int_{x_{0}}^{y} \eta^{*}(z)(y-z) d z=\psi(y)-y \psi^{\prime}\left(x_{0}+\right)-\int_{x_{0}}^{y} \psi^{\prime \prime}(z)(y-z) d z=\psi\left(x_{0}\right)-x_{0} \psi^{\prime}\left(x_{0}+\right)$.

\section{References}

[1] J. Azéma and M. Yor. Une solution simple au problème de Skorokhod. In Séminaire de Probabilités XIII, volume 721, pages 90-115. Springer Berlin Heidelberg, 1979.

[2] Suleyman Basak and Dmitry Makarov. Strategic asset allocation in money management. Journal of Finance, Forthcoming, May 2012.

[3] D. G. Hobson and J. L. Pedersen. The minimum maximum of a continuous martingale with given initial and terminal laws. Ann. Probab., 30(2):978999, 2002.

[4] Robert Kertz and Uwe Rösler. Martingales with given maxima and terminal distributions. Israel Journal of Mathematics, 69:173-192, 1990. 10.1007/BF02937303.

[5] E. Perkins. The Cereteli-Davis solution to the $H^{1}$-embedding problem and an optimal embedding in brownian motion. Seminar on stochastic processes, 1985, pages 173-223, 1986.

[6] Christian Seel and Philipp Strack. Gambling in contests. Discussion Paper Series of SFB/TR 15 Governance and the Efficiency of Economic Systems 375, Mar 2012.

[7] A.V. Skorokhod. Studies in the theory of random processes. Addison-Wesley, Reading, Mass., 1965. 